\title{
Off-resonance Effect
}

National Cancer Institute

\section{Source}

National Cancer Institute. Off-resonance Effect. NCI Thesaurus. Code C86997.

An artifact resulting from a signal which has a frequency different from that expected for the system. 